\title{
Correcting the beam centroid motion in an induction accelerator and reducing the beam breakup instability
}

\author{
J. E. Coleman, C. A. Ekdahl, D. C. Moir, G. W. Sullivan, and M. T. Crawford \\ Los Alamos National Laboratory, Los Alamos, New Mexico 87545, USA
}

(Received 9 February 2014; published 15 September 2014)

\begin{abstract}
Axial beam centroid and beam breakup (BBU) measurements were conducted on an 80 ns FWHM, intense relativistic electron bunch with an injected energy of 3.8 MV and current of $2.9 \mathrm{kA}$. The intense relativistic electron bunch is accelerated and transported through a nested solenoid and ferrite induction core lattice consisting of 64 elements, exiting the accelerator with a nominal energy of $19.8 \mathrm{MeV}$. The principal objective of these experiments is to quantify the coupling of the beam centroid motion to the BBU instability and validate the theory of this coupling for the first time. Time resolved centroid measurements indicate a reduction in the BBU amplitude, $\langle\xi\rangle$, of $19 \%$ and a reduction in the BBU growth rate $(\Gamma)$ of $4 \%$ by reducing beam centroid misalignments $\sim 50 \%$ throughout the accelerator. An investigation into the contribution of the misaligned elements is made. An alignment algorithm is presented in addition to a qualitative comparison of experimental and calculated results which include axial beam centroid oscillations, BBU amplitude, and growth with different dipole steering.
\end{abstract}

DOI: 10.1103/PhysRevSTAB.17.092802

PACS numbers: 29.27.Bd, 52.59.Sa, 41.75.Ht, 29.20.Ej

\section{INTRODUCTION}

Relativistic particle accelerators are highly sensitive to centroid offsets because of the multiple elements used for transport and acceleration in the accelerator lattice. These centroid offsets can lead to emittance growth and contribute to the beam breakup (BBU) instability or wakefield effects [1-7]. The BBU instability, a transverse magnetic coupling which destroys the beam quality, was discovered by the authors of Refs. [8,9] and has been studied extensively in both rf and induction accelerators [5,6,10-20]. The Advanced Test Accelerator demonstrated the highest intensity transport in an induction accelerator to date with a $10 \mathrm{kA}, 50 \mathrm{MeV}$ electron beam utilizing phase mix damping through a laser ionized channel to suppress the BBU $[12,13,21]$. A summary of these previous BBU studies and recent increased intensity and vacuum transport on an induction accelerator with 64 cells is described in Ref. [22].

Centroid offsets can lead to large emittance growth; this has been examined analytically for the International Linear Collider (ILC) by several authors over the last decade [1-4]. References [1,2] estimated $\Delta \epsilon / \epsilon \sim 25 \%$ after 50 FODO (periodic focusing) cells of quadrupole transport. Reference [1] used a betatron mismatch injection error to initiate the emittance growth. Reference [2] initiated the growth with $100 \mu \mathrm{m}$ quadrupole misalignments and random ground motion over long periods of time. References [3,4] analyzed the contribution of centroid

Published by the American Physical Society under the terms of the Creative Commons Attribution 3.0 License. Further distribution of this work must maintain attribution to the author $(s)$ and the published article's title, journal citation, and DOI. offsets to emittance growth as a function of energy for $1 \mu \mathrm{m}$ quadrupole offset, $15 \mu \mathrm{rad}$ accelerator cavity tilt, and $50 \mu \mathrm{m}$ correction errors due to beam position monitor (BPM) offsets. Accelerator cavity tilt lead to a linear increase in emittance with respect to increased energy and the quadrupole offset lead to a much more rapid increase with respect to increased energy.

Beams with intense space charge face a slightly different challenge. The number of elements in existing induction linacs is comparable to a single pass in the ILC. The transport magnetic field used in induction linacs is slightly higher, but the rigidity $[B \rho]$ of the beams is $>10^{3} \times$ lower than the ILC which contributes to $>1 \mathrm{~mm}$ centroid offsets in the beam distribution. Centroid offsets on DARHT Axes I\&II are $\sim 5 \mathrm{~mm}$ throughout the whole accelerator and have been estimated to contribute $<4 \%$ emittance growth [7]. The pulse lengths or bunch lengths of these beams are $>10^{3} \times$ longer. Energy variation from head to tail and misalignments over these pulses leads to corkscrew, a time dependent centroid motion due to chromaticity [23-25].

Estimates of the contribution of displacement and rotational (angular) offsets of the focusing elements to the beam centroid offsets were made for a nonrelativistic space charge dominated $K^{+}$beam in a 4 solenoid lattice $[26,27]$. Assuming random displacement and rotational offsets of $1.5 \mathrm{~mm}$ and $5 \mathrm{mrad}$ of each of the 4 solenoids, a total contribution $\sim 3 \mathrm{~mm}$ and $\sim 8 \mathrm{mrad}$ was calculated at the exit of the transport lattice [27]. Reference [26] also proposed an alignment algorithm utilizing two dipole pairs in which a $4 \times 4$ matrix is inverted and requires a minimum of five measurements. These dipole correction measurements were demonstrated in Refs. [27,28]. Misalignments of the emitter and the solenoid lattice contributed to 
nonlinear focusing effects resulting in a nonuniform $J(x, y)$ [29] and an ensemble of lattice iterations demonstrated this effect in Ref. [28].

The first axis of the Dual-Axis Radiography for Hydrodynamic Testing (DARHT) facility [22] is exploring the limitations of increasing the intensity of the electron beam for future radiographic capabilities. After increasing the current by $70 \%$ on DARHT Axis-I it was determined that the beam centroid was substantially offset and needed to be corrected for transport of the beam to the target. In order to effectively increase the intensity of the beam, the coupling of the beam centroid to BBU must be fully understood and reduced. The results presented below provide the first successful demonstration of the reduction of $\mathrm{BBU}$ in an induction accelerator through the correction of the beam centroid and full vacuum transport through 64 cells without disruption of the beam quality. These results also provide the first independent validation of the theory which explains the coupling of the beam centroid to the BBU instability. The rms centroid was reduced by $50 \%$ through the use of a pair of nested dipoles at several $z$ locations within the accelerator lattice; as a result a $19 \%$ reduction in the average BBU amplitude, $\langle\xi\rangle$, and a $4 \%$ reduction in the growth rate, $\Gamma$, was also observed.

\section{BEAM MISALIGNMENTS AND BBU INSTABILITY}

As stated in Ref. [22], BBU manifests itself as a transverse magnetic coupling to destroy the beam quality. The BBU growth along the accelerator is characterized by the equation below [30]:

$$
\frac{\xi}{\xi_{o}}=\left(\frac{\gamma_{o}}{\gamma}\right)^{1 / 2} \exp \left(\Gamma_{m}\right)
$$

where $\xi$ is the measured BBU amplitude at a given location and $\xi_{o}$ is the measured BBU amplitude at the entrance of the accelerator. The amplitude decreases with acceleration to $1 / 2$ power and increases exponentially with the maximum growth factor, $\Gamma_{m}$ :

$$
\Gamma_{m}=\frac{1}{c} I_{b} N_{g} Z_{\perp}\left\langle\frac{1}{B}\right\rangle,
$$

where $c$ is the speed of light, $I_{b}(A)$ is the beam current, $N_{g}$ is the number of gaps, $Z_{\perp}(\Omega / m)$ is the transverse impedance of the induction cell cavity, and $\langle 1 / B\rangle(1 / T)$ is the average of the inverse magnetic field strength.

The impact the beam centroid offset has on the BBU instability is nested in the transverse coupling impedance, $Z_{\perp}(\Omega)$, of the cells as calculated with the formula, first derived by Refs. [30,31]

$$
\frac{Z_{\perp}}{Q}=\frac{\left(c \int B_{y} d z\right)^{2}}{2 \omega_{o} U},
$$

where $Q$ is the quality factor of the cell cavity at the resonant frequency, $B_{y} d z$ is the transverse magnetic field component which imparts change in the transverse momentum to the particles as they traverse the acceleration gap, $d z$, $\omega_{o}$ is the resonant frequency, and $U$ is the stored energy in the cell. The transverse magnetic field component can also be written as a transverse voltage:

$$
V_{t}=c \int B_{y} d z
$$

The coupling of the beam centroid motion to the BBU instability is rooted in the $\mathrm{TM}_{1 n 0}$ modes in the induction cell, which are driven by a displaced current, $I$, with a centroid offset of $\langle r\rangle$ as shown in the transverse voltage formulation:

$$
V_{t}=-\frac{i}{c} \omega_{o} Z_{\perp} I\langle r\rangle .
$$

This formulation indicates the importance of minimizing the beam centroid offset. An estimate of the transverse voltage induced by a $2.9 \mathrm{kA}$ beam with $\langle r\rangle=1 \mathrm{~mm}$ in a DARHT Axis-I cell gap with $Z_{\perp}=1 \mathrm{k} \Omega$ and $\omega_{o}=4.4 \times$ $10^{9} \mathrm{rad} / \mathrm{s}$ is $43 \mathrm{kV}$. This is $17 \%$ of axial voltage provided by the cell to accelerate the beam. The centroid offset creates the transverse magnetic field component, $B_{y} d z$, driving the $\mathrm{TM}_{1 n 0}$ mode and initiating the $\mathrm{BBU}$, or $\mathrm{rf}$ oscillation on the beam envelope. Once initiated, the BBU centroid oscillation continues to drive the $\mathrm{TM}_{1 n 0}$ mode in advancing cavities, which leads to the growth rate of BBU as indicated by Eqs. (1)-(5). As will be shown below in Sec. VII reducing the beam centroid motion along the accelerator by $50 \%$ reduced $\langle\xi\rangle$ by $19 \%$ and $\Gamma_{m}$ by $4 \%$.

\section{EXPERIMENTAL SETUP}

The experimental configuration used to study the correction of the beam centroid and its coupling to the BBU instability was the DARHT Axis-I linear induction accelerator (Fig. 1) [22]. The accelerator is composed of a 3.8 MV injector and 64, $250 \mathrm{kV}$ induction cells are used to accelerate the beam to an end point energy of $19.8 \mathrm{MeV}$. Each induction cell has a nested solenoid and an $x$ and $y$ dipole pair. A minimum number of dipoles were used to correct the beam centroid along the length of the accelerator.

Misalignments in induction accelerators are contributed to nine separate offsets: displacement and rotational offsets in the diode, beam position monitors, accelerator cells and solenoids, and finally a rotational offset of the emitter. Including all of the BPMs, cells, and magnets from source to target there are 319 individual offsets in this accelerator. Below we will discuss the contributions of the dipoles to beam alignment and the solenoids and induction cells to beam misalignment. 


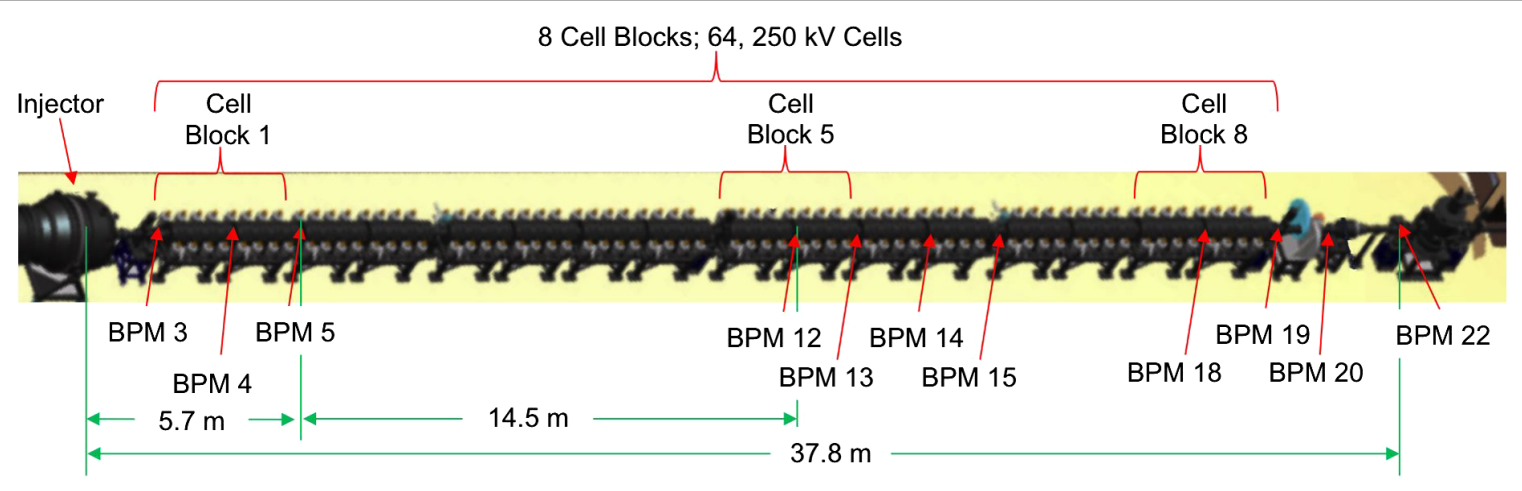

FIG. 1. Model of the DARHT Axis-1 accelerator, consisting of the $4 \mathrm{MV}$ injector, 64 induction cells, and a BPM located after every 4 cells.

\section{A. Dipoles}

The nested dipoles used on DARHT Axis-I are simply current sheets built onto printed circuit board. The first set of dipoles, the anode dipoles $[A(x, y)]$ are built into the first transport magnet, or the anode magnet, and the second set, the matching dipoles $[M(x, y)]$ are a few $\mathrm{cm}$ downstream of the center line of the anode magnet. Together these two dipole pairs are used to fully correct the beam centroid as it enters the accelerator lattice. $A(x, y)$ and $M(x, y)$ have an effective length of $21.6 \mathrm{~cm}$ and a radius of $6.5 \mathrm{~cm}$. The equivalent field strength is $2.7 \mathrm{G} / \mathrm{A}$. The maximum current for these magnets is limited to $10 \mathrm{~A}$ or a field of $27 \mathrm{G}$. In order to determine the kick provided by a dipole magnet, one must use the strength parameter derived from the envelope equation $[26,32,33]$ :

$$
S=\frac{B_{r}}{[B \rho]},
$$

where $B_{r}$ is the radial magnetic field and $[B \rho]$ is the rigidity of the beam:

$$
[B \rho]=\frac{1}{q} \gamma \beta c m_{e}
$$

where $q$ is the electron charge, $\gamma$ is the Lorentz factor, $\beta=v / c$ or the ratio of the beam velocity to the speed of light, $c$, and $m_{e}$ is the electron mass. $27 \mathrm{G}$ is sufficient to kick the $3.8 \mathrm{MeV}$ electron beam, $[B \rho]=1.4 \times 10^{-2} \mathrm{~T}-\mathrm{m}$, up to $40 \mathrm{mrad}$ at the center of the dipole.

There is a pair of dipoles nested in every solenoid in the transport lattice. These cell steering magnets have an effective length of $15.6 \mathrm{~cm}$, radius of $5.1 \mathrm{~cm}$, and an equivalent field strength of $1.8 \mathrm{G} / \mathrm{A}$. The maximum current for these magnets is limited to $5 \mathrm{~A}$ or a field of $9.2 \mathrm{G}$. The beam energy throughout the accelerator cells ranges from 3.8-19.8 MeV, or $[B \rho]=1.4-6.7 \times 10^{-2} \mathrm{~T}-\mathrm{m}$, and $9.2 \mathrm{G}$ is sufficient to kick low energy electrons up to $10 \mathrm{mrad}$ at the center of the dipole and high energy electrons only $2 \mathrm{mrad}$.

\section{B. Solenoid magnets}

After the electron beam is extracted through the diode it is initially matched by the first transport magnet, or the anode magnet. The effective length of the anode magnet is $46.9 \mathrm{~cm}$, radius of $10.1 \mathrm{~cm}$, and the equivalent field strength is $3.5 \mathrm{G} / \mathrm{A}$. The maximum current for this magnet is near $250 \mathrm{~A}$, although our tunes typically require $200 \mathrm{~A}$, or a field of $700 \mathrm{G}$ to match the beam with the desired envelope parameters for accelerator transport. Assuming displacement and rotational offsets of $1 \mathrm{~mm}$ and $5 \mathrm{mrad}$, this solenoid can lead to beam offsets of $1 \mathrm{~mm}$ and $5 \mathrm{mrad}$ at the entrance to the accelerator.

The beam is then transported through the accelerator that consists of 64 more solenoids nested amongst the induction cells. Each of these magnets has an effective length of $29.8 \mathrm{~cm}$ and a radius of $9.8 \mathrm{~cm}$. The current required for these magnets increases linearly along the length of the accelerator and depends on the beam space charge, or the cathode being used. Examining the $2.9 \mathrm{kA}$ beam presented here the solenoid strength ranges from $0.4-2 \mathrm{kG}$ for our minimum BBU tune [22]. A $1 \mathrm{~mm}$ position offset is equivalent to an angular displacement $>6 \mathrm{mrad}$ about the center of a $30-\mathrm{cm}$-long solenoid.

Displacement and rotational offsets of $1 \mathrm{~mm}$ and $5 \mathrm{mrad}$ for each transport magnet could lead to beam centroid offsets that range from 1-5 $\mathrm{mm}$ and 5-25 mrad throughout the accelerator. Combining these contributions along the accelerator could lead to substantial alignment issues. The rotational offset is the largest contributor and as the solenoid current in the accelerator is increased the offset contribution becomes larger and alignment becomes more critical.

The displacement and rotational offsets and their contribution to beam centroid misalignments are a function of the strength parameters of the solenoids derived from the envelope equation $[26,32,33]$ :

$$
S=\frac{B}{[B \rho]},
$$


where $B$ is the magnetic flux density in the solenoid in Tesla and $[B \rho]$ is the rigidity of the beam defined above. The strength parameter indicates the dependence on the magnetic field and beam energy. Moderate energy beams with $\gamma<100$ and high space charge, like DARHT Axis-I, require large $B(>1 \mathrm{kG})$ for transport and therefore are more susceptible to centroid offsets $>1 \mathrm{~mm}$.

\section{Induction cells}

Once the electron beam is matched by the first transport magnet and cell magnet it traverses the first induction cell gap. The Axis-I induction cell design is described in Refs. [22,34]. Each cell consists of a ferrite induction core that is driven with an oil-insulated transmission line. The displacement and rotational offsets and their contribution to beam centroid misalignments are a function of the strength parameters of the induction cells derived from the envelope equation $[26,32,33]$ :

$$
S=\frac{\Phi}{\gamma \beta^{3} c[B \rho]} \frac{\pi}{L},
$$

where $\Phi$ is the acceleration voltage applied across the induction cell gap, which is typically $250 \mathrm{kV}$, and $L$ is the accelerating gap length, which is $19 \mathrm{~mm}$. This formulation indicates a $1 \mathrm{~mm}$ cell offset on the first cell kicks the $3.8 \mathrm{MeV}$ beam, $[B \rho]=1.4 \times 10^{-2} \mathrm{~T}-\mathrm{m}$, about 2 mrad. Once $\gamma>12$, after the first cell block, the kick $<1 \mathrm{mrad}$ and becomes negligible with increasing $\gamma$. Tilts due to the induction cells can be ignored because the acceleration gap is only $19 \mathrm{~mm}$ providing a minimal dipole kick. The solenoid magnets and dipoles have a larger contribution to the beam centroid offset, particularly at lower energy.

\section{CENTROID MEASUREMENTS}

Our standard tune for the $1.7 \mathrm{kA}$ beam was initially used to transport the $2.9 \mathrm{kA}$ beam [Fig. 2(a)]. The envelope plot in Fig. 2(b) indicates the $2.9 \mathrm{kA}$ beam has an envelope at least $50 \%$ larger than the $1.7 \mathrm{kA}$ beam. The measured rms centroid, $\langle r\rangle$, along the accelerator is also shown for the two current amplitudes in Fig. 2(c). These were averaged over five shots for each case and the error bars indicate the variation is within the resolution of the measurements. Position by position the average $2.9 \mathrm{kA}$ beam centroid offset is $>60 \%$ larger and over the full length of the accelerator $\langle r\rangle=2.67 \pm 1.25 \mathrm{~mm}$ versus $\langle r\rangle=1.95 \pm 1.20 \mathrm{~mm}$ for the $1.7 \mathrm{kA}$ beam.

We examined the $2.9 \mathrm{kA}$ beam more closely by calculating the betatron oscillations of the beam centroid along the accelerator lattice with our XTR envelope code $[35,36]$ using the initial centroid conditions measured at BPM03, $\langle x\rangle=-2.23 \mathrm{~mm}$ and $\langle y\rangle=0.41 \mathrm{~mm}$ in Fig. 3(a). These calculations indicate there are eight betatron oscillations of the beam centroid throughout the accelerator lattice. It is also evident from Fig. 3(a) that the betatron wavelength grows axially, which is expected because the average $B$ field in the lattice remains relatively constant for $z>10 \mathrm{~m}$ while $\gamma$ is increasing at a rate of $0.96 / \mathrm{m}$. With these initial position offsets and no angular contribution, the calculated beam centroid has a maximum oscillation of $\pm 0.7 \mathrm{~mm}$. The calculated beam centroid in the lab frame does not perfectly encircle the magnetic axis because it is launched with an initial offset in the fringe field of a solenoid. Similar to single particle motion, the centroid will gyrate about a flux tube along the magnetic lattice. This is also shown analytically by Refs. [6,37].

In the experiment we measure a small angular offset relative to the BPM measurement axis; in the $x$ direction the angle is $+0.08 \mathrm{mrad}$ and in the $y$ direction the angle is $-0.10 \mathrm{mrad}$ as shown by the fit to the curves in Fig. 3(b). The addition of an initial $3 \mathrm{mrad}$ angular offset to the calculation in Fig. 3(a) roughly produces the same offset growth in Fig. 3(b). We normalize out the angle and the mean offset of the two curves in Fig. 3(b) to determine if we are resolving the betatron motion of the beam centroid with respect to this angle [Fig. 3(c)]. The $\sigma$ of these normalized centroid offsets are $\langle x\rangle= \pm 0.72 \mathrm{~mm}$ and
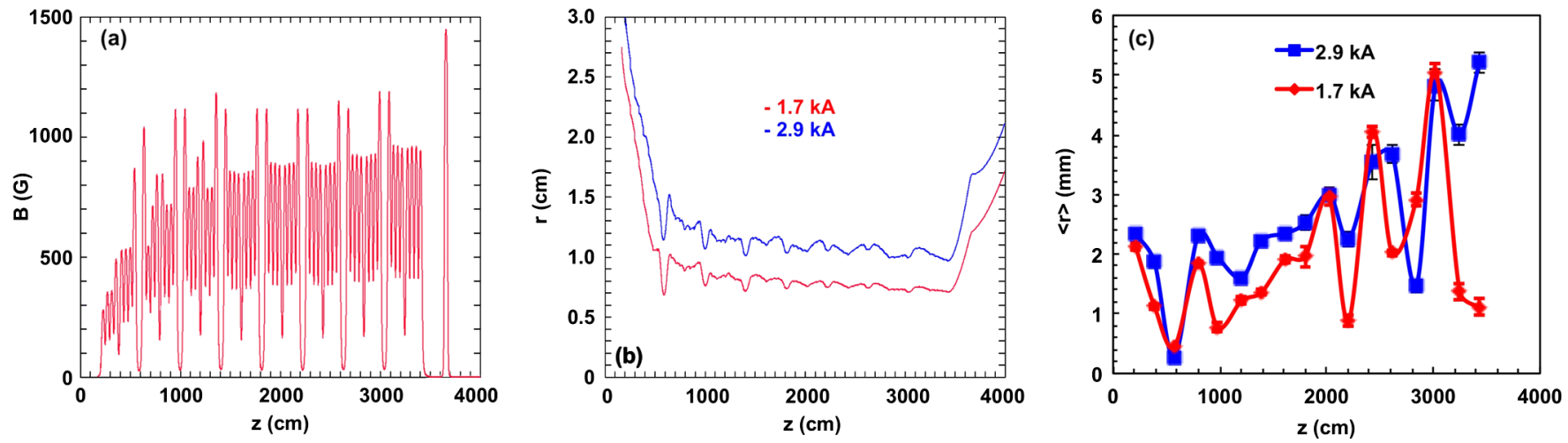

FIG. 2. (a) Solenoid lattice for tune used to transport the $1.7 \mathrm{kA}$ beam, (b) calculated envelope comparison between the nominal $1.7 \mathrm{kA}$ beam (red) and the $2.9 \mathrm{kA}$ beam (blue), and (c) measured rms beam centroid offsets, $\langle r\rangle$, for both current amplitudes. 

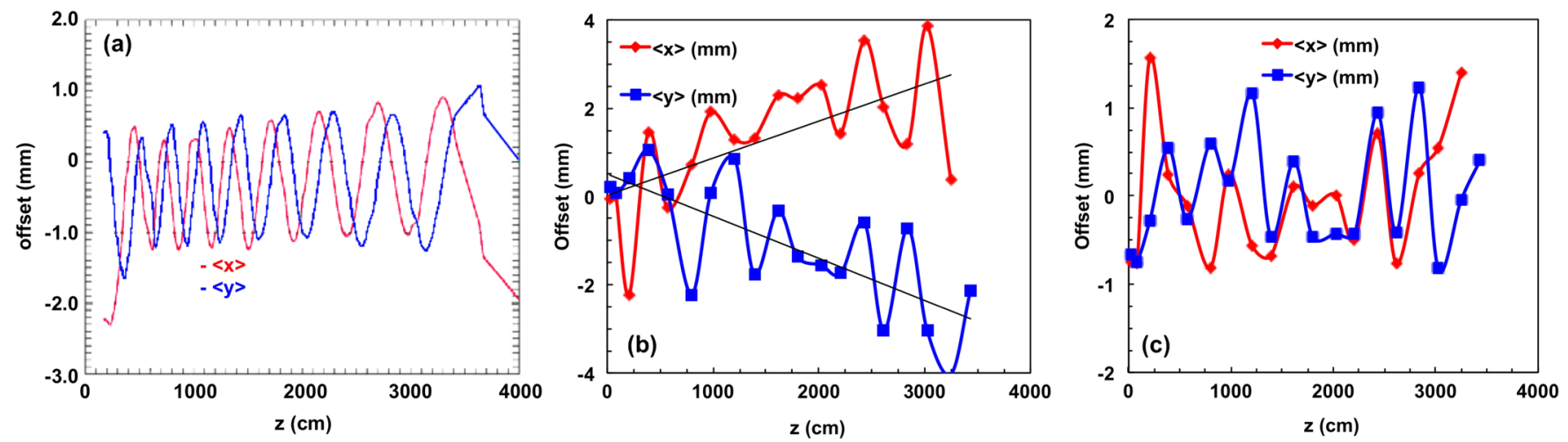

FIG. 3. (a) Calculated beam centroid oscillations corresponding to the $2.9 \mathrm{kA}$ tune with initial position offsets only, (b) measured beam centroid offsets running this tune, (c) normalized measured betatron oscillation of the beam centroid (note scale differences).

$\langle y\rangle= \pm 0.65 \mathrm{~mm}$, close to the maximum excursions calculated with XTR. This data indicates we nearly resolve the betatron oscillations of the beam centroid for an accelerator tune with this magnetic field strength. This is because our BPMs have axial spacings of $185-224 \mathrm{~cm}$ and the betatron wavelength, $\lambda_{\beta}$, for this particular tune ranges from $250 \mathrm{~cm}$ at the upstream end of the accelerator to $550 \mathrm{~cm}$ near the end.

\section{ALIGNMENT METHOD}

Increasing the beam intensity by $70 \%$ led to a $50 \%$ increase in the beam envelope and $>30 \%$ increase in $\langle r\rangle$ as shown in the previous section; these centroid offsets needed to be reduced in order to transport the beam to the target. The desired centroid offsets for beams with these intensities in an induction accelerator lattice are $\sim 1 \mathrm{~mm}$ and $\sim 1 \mathrm{mrad}$. However when examining the alignment of the beam with the nominal steering used at the entrance and the end of the accelerator for the $2.9 \mathrm{kA}$ beam the rms centroid offsets ranged from $\langle r\rangle=2 \pm 1.2 \mathrm{~mm}$ as will be shown below.

An alignment algorithm developed by Ref. [26] [Eq. (10)] was employed for corrections. The algorithm calculates the correct current values for dipoles $N_{x}, N_{y}$, $M_{x}$, and $M_{y}$, the first term in Eq. (10), utilizing the initial dipole currents, the second term, the $4 \times 4$ transfer matrix, $D$, which is outlined in Eqs. (11) and (12), and the difference between the initial centroid offsets and the desired centroid offsets of zero. The transfer matrix, $D$, is generated by incrementally making a single iteration from the initial settings with one dipole magnet of the 2-dipole pairs of choice to correct the beam centroid $\langle x\rangle,\langle y\rangle,\left\langle x^{\prime}\right\rangle$ and $\left\langle y^{\prime}\right\rangle$, at a single location. This requires five separate measurements, which provide a large enough excursion on the beam centroid utilizing the minimum dipole current, $d\langle x\rangle / d I$, without scraping the beam. In practice this may require 10 measurements to provide an accurate data set,

$$
\left[\begin{array}{l}
N_{x} \\
N_{y} \\
M_{x} \\
M_{y}
\end{array}\right]=\left[\begin{array}{l}
N_{x i} \\
N_{y i} \\
M_{x i} \\
M_{y i}
\end{array}\right]-D^{-1}\left[\left[\begin{array}{c}
\left\langle x_{i}\right\rangle \\
\left\langle x_{i}^{\prime}\right\rangle \\
\left\langle y_{i}\right\rangle \\
\left\langle y_{i}^{\prime}\right\rangle
\end{array}\right]-\left[\begin{array}{l}
0 \\
0 \\
0 \\
0
\end{array}\right]\right],
$$

where

$$
D=\left[\begin{array}{llll}
A_{x y \_} i & B_{x y_{-} i} & C_{x y_{-} i} & D_{x y_{-} i}
\end{array}\right]
$$

and

$$
\begin{gathered}
A_{x y_{-} i}=\left[\begin{array}{l}
\frac{d\langle x\rangle}{d I N_{x}} \\
\frac{d\left\langle x^{\prime}\right\rangle}{d I N_{x}} \\
\frac{d\langle y\rangle}{d I N_{x}} \\
\frac{d\left\langle y^{\prime}\right\rangle}{d I N_{x}}
\end{array}\right], \quad B_{x y_{-} i}=\left[\begin{array}{l}
\frac{d\langle x\rangle}{d I N_{y}} \\
\frac{d\left\langle x^{\prime}\right\rangle}{d I N_{y}} \\
\frac{d\langle y\rangle}{d I N_{y}} \\
\frac{d\left\langle y^{\prime}\right\rangle}{d I N_{y}}
\end{array}\right], \\
C_{x y_{-} i}=\left[\begin{array}{l}
\frac{d\langle x\rangle}{d I M_{x}} \\
\frac{d\left\langle x^{\prime}\right\rangle}{d I M_{x}} \\
\frac{d\langle y\rangle}{d I M_{x}} \\
\frac{d\left\langle y^{\prime}\right\rangle}{d I M_{x}}
\end{array}\right], \quad D_{x y_{-} i}=\left[\begin{array}{l}
\frac{d\langle x\rangle}{d I M_{y}} \\
\frac{d\left\langle x^{\prime}\right\rangle}{d I M_{y}} \\
\frac{d\langle y\rangle}{d I M_{y}} \\
\frac{d\left\langle y^{\prime}\right\rangle}{d I M_{y}}
\end{array}\right] .
\end{gathered}
$$

Position measurements are made at every BPM; however, the angles are not. In order to properly employ these measurements we propagated the beam in "nearly" a fieldfree drift and measured the centroid offsets with an upstream and downstream BPM. We say "nearly" field free because the transformer coupling of the induction cells to the beam effectively reduces the beam energy. The beam creates a load impedance on the induction cells of $13.8 \Omega$. The $2.9 \mathrm{kA}$ beam loads down each induction cell by $\sim 40 \mathrm{kV}$; this is shown for the drifting beam in cells 5-12, where we accelerated the beam through the injector and the first four cells only; and cells 45-52 where we only accelerated the beam with five cell blocks (Fig. 4). $40 \mathrm{kV}$ is only $1 \%$ of the beam energy downstream of the injector and $<0.3 \%$ after cell block 5 (CB5). These cell 

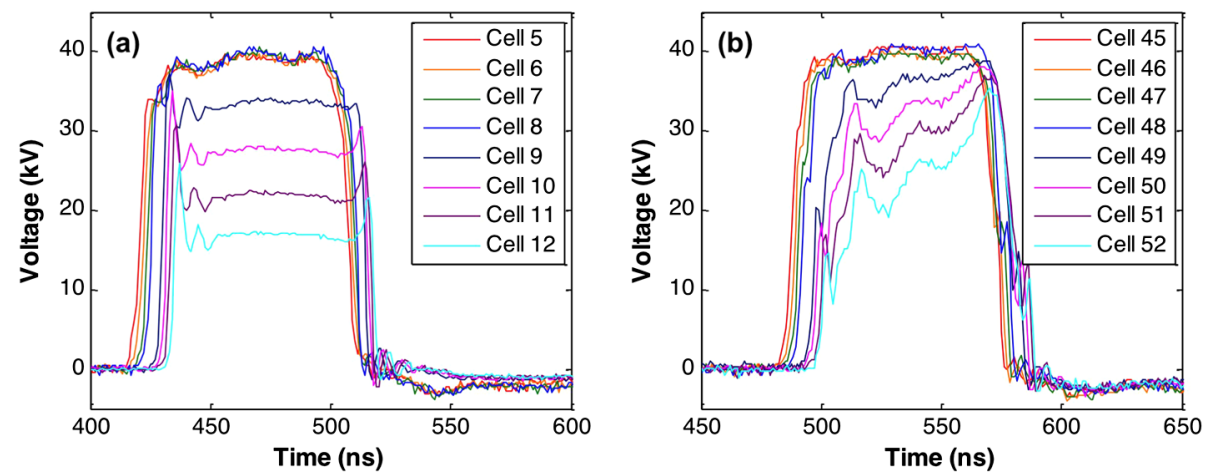

FIG. 4. Measured cell loading by the $2.9 \mathrm{kA}$ beam on induction (a) cells 5-12 for shot 16691 and; (b) cells $45-52$ for shot 16949 .

voltage monitors provide additional confirmation of the beam loading and the expansion of the envelope due to space-charge forces. The beam loading is reduced after cell 8 in Fig. 4(a) and after cell 48 in Fig. 4(b) because the beam has begun to scrape and lose current, indicating the restrictions on using downstream BPMs for the alignment measurements described below.

\section{ALIGNMENT MEASUREMENTS}

The alignment method mentioned in the previous section was used to steer the beam for a separate higher field tune, tune 6. A step-by-step procedure was used to align the beam along the accelerator. First we aligned the beam at the upstream end of the accelerator in the first cell block with dipoles $A(x, y)$ and $M(x, y)$. As centroid offsets began to grow $>2 \mathrm{~mm}$ after CB5 we performed another alignment iteration with dipoles $33(x, y)$ and $36(x, y)$. Finally we aligned the beam at the end of the accelerator with dipoles $57(x, y)$ and $60(x, y)$. The results are described in the subsections below.

Tune 6 was developed to help reduce the BBU amplitude by $5 \times$ with a $20 \%$ increase in $\langle B\rangle$ from Tune 4 [22]
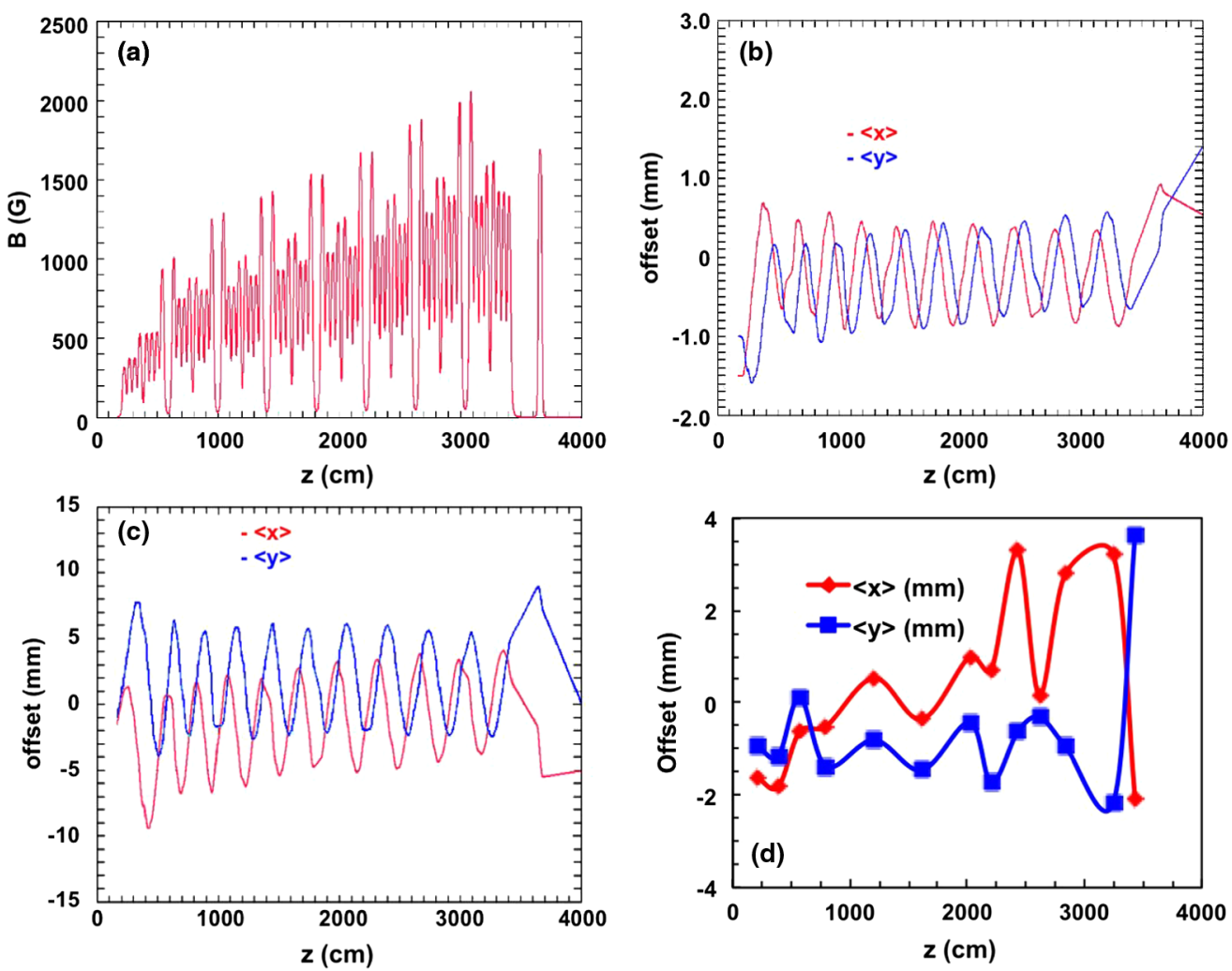

FIG. 5. (a) Solenoid lattice for a higher field tune, tune 6, for the $2.9 \mathrm{kA}$ beam; (b) calculated beam centroid oscillations corresponding to this tune with initial position offsets only; (c) calculated beam centroid oscillations corresponding to this tune with initial position and assumed angle offsets of $5 \mathrm{mrad}$; (d) measured beam centroid offsets running this tune (note scale differences). 

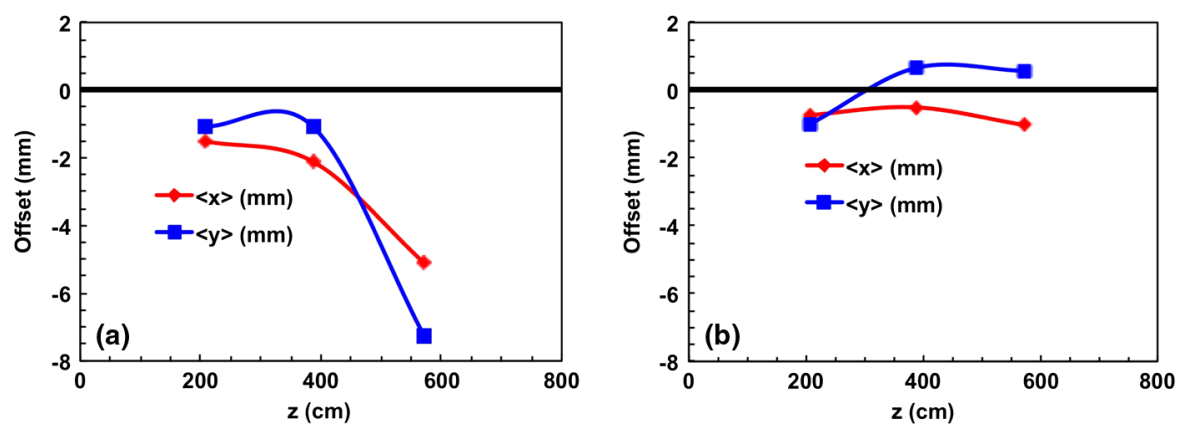

FIG. 6. Measured beam centroid offsets with (a) initial dipole settings for the uncorrected case; (b) corrected centroid offset. Each set was measured only operating the first four induction cells and their nested solenoids with tune 6 .

[Figs. 2(a) and 5(a)]. The number of betatron oscillations of the beam centroid for this tune is 10 as seen in Fig. 5(b) using the initial centroid offsets measured at BPM03 of $\langle x\rangle=-1.5 \mathrm{~mm}$ and $\langle y\rangle=-1 \mathrm{~mm}$ only. The maximum excursion of the centroid varies $\pm 0.7 \mathrm{~mm}$ and $\lambda_{\beta}$ ranges from $250-460 \mathrm{~cm}$. As stated above, the calculated beam centroid in the lab frame does not perfectly encircle the magnetic axis because it is launched with an initial offset in the fringe field of a solenoid. The addition of a $5 \mathrm{mrad}$ angular offset to the initial beam centroid [Fig. 5(c)] increases the calculated maximum excursion of the centroid throughout the accelerator $>5 \times$ to $\pm 4 \mathrm{~mm}$, close to what is measured in Fig. 5(d), particularly in the downstream section. It is also worth noting in the calculation that the axis of gyration of the beam centroid is $>2 \mathrm{~mm}$ away from the magnetic axis and is reduced slightly along the accelerator lattice with ideal magnet alignment. During the experimental setup at this time we were not sampling the beam centroid on BPMs 7, 9, and 11. The betatron oscillations of the beam centroid increase linearly with the magnetic field; this explains the difficulty of being able to resolve the betatron oscillations with this higher field tune. To resolve the betatron motion of the beam centroid we need to sample at $f>$ Nyquist, or BPM spacing $<\lambda_{\beta} / 2$; for this tune it would require a BPM every 2 cells.

\section{A. Injector steering}

Initially the beam misalignment upon the entrance to the accelerator lattice, BPM03, was manageable. However, the offsets grew axially along the accelerator [Fig. 5(d)], so we began steering the beam centroid where centroid offsets were $>2 \mathrm{~mm}$. In order to understand the origination of these offsets we decided to simplify the problem and only operate the first four induction cells and their nested solenoids. This provided useful insight, at the exit of the fourth solenoid, BPM04, where we measured a centroid offset of $\langle x\rangle=-2.1 \mathrm{~mm}$ and $\langle y\rangle=-1.1 \mathrm{~mm}$ [Fig. 6(a)]. We were also able to extrapolate the angle, $\left\langle x^{\prime}\right\rangle=$ $-1.6 \mathrm{mrad}$ and $\left\langle y^{\prime}\right\rangle=-3.3 \mathrm{mrad}$, from the measured centroid offset at BPM05 assuming a field-free drift (Table I). We say field free as explained above, because we are extracting $40 \mathrm{kV}$ of energy from the beam per cell for a total of $160 \mathrm{kV}$ through cells 5-8. This can be ignored to first order because $160 \mathrm{kV}$ is only $4 \%$ of the total kinetic energy of the beam, which has a small impact on the beam envelope and centroid. Beyond BPM05 the beam distribution was $>5 \mathrm{~cm}$ and offset $>1 \mathrm{~cm}$, so no centroid measurements were taken downstream with any confidence due to beam scraping.

The growth of the position and angle offsets downstream of BPM04 [Figs. 5(d) and 6(a)] provided motivation for alignment. We performed single dipole iterations using the anode magnet and matching dipoles $(A(x, y)$ and $M(x, y))$ and inverted the $4 \times 4$ matrix to determine the correct settings for $A(x, y)$ and $M(x, y)$. The corrected beam centroid up to BPM05 is shown in Fig. 6(b) and Table I.

\section{B. Cell block 5 steering}

Despite improving the centroid offsets in the first cell block, as we worked our way down the accelerator, the beam centroid continued to oscillate about the ideal $z$ axis and seemed to be growing largely out of control in the $x$ direction after the CB5, at BPM14 ( $z=24.3 \mathrm{~m})$ [Fig. 7(a)]. This is understandable because the magnetic field is increasing linearly along the axis as shown in Fig. 5(a) and this increases the strength parameter [Eq. (8)], which contributes to the position and angle offsets from each solenoid. In the interest of time and for practicality we only corrected the beam centroid where it began to get largely out of control or where $\langle r\rangle>2 \mathrm{~mm}$ and $\left\langle r^{\prime}\right\rangle>2 \mathrm{mrad}$, where $\langle r\rangle$ and $\left\langle r^{\prime}\right\rangle$ are the rms position and angle. So, we isolated the problem again and only ran the first five cell blocks and began measuring corrections in CB5 and downstream.

TABLE I. Measured beam centroid correction after 4 cells at BPM04 $(z=3.87 \mathrm{~m})$ using dipoles $A(x, y)$ and $M(x, y)$.

\begin{tabular}{lcccccc}
\hline \hline & Shot & $\begin{array}{c}z \\
(\mathrm{~cm})\end{array}$ & $\begin{array}{c}\langle x\rangle \\
(\mathrm{mm})\end{array}$ & $\begin{array}{c}\left\langle x^{\prime}\right\rangle \\
(\mathrm{mrad})\end{array}$ & $\begin{array}{c}\langle y\rangle \\
(\mathrm{mm})\end{array}$ & $\begin{array}{c}\left\langle y^{\prime}\right\rangle \\
(\mathrm{mrad})\end{array}$ \\
\hline Uncorrected & 16939 & 387 & -2.15 & -1.60 & -1.11 & -3.32 \\
Corrected & 16940 & 387 & -0.52 & -0.26 & 0.67 & -0.04 \\
\hline \hline
\end{tabular}



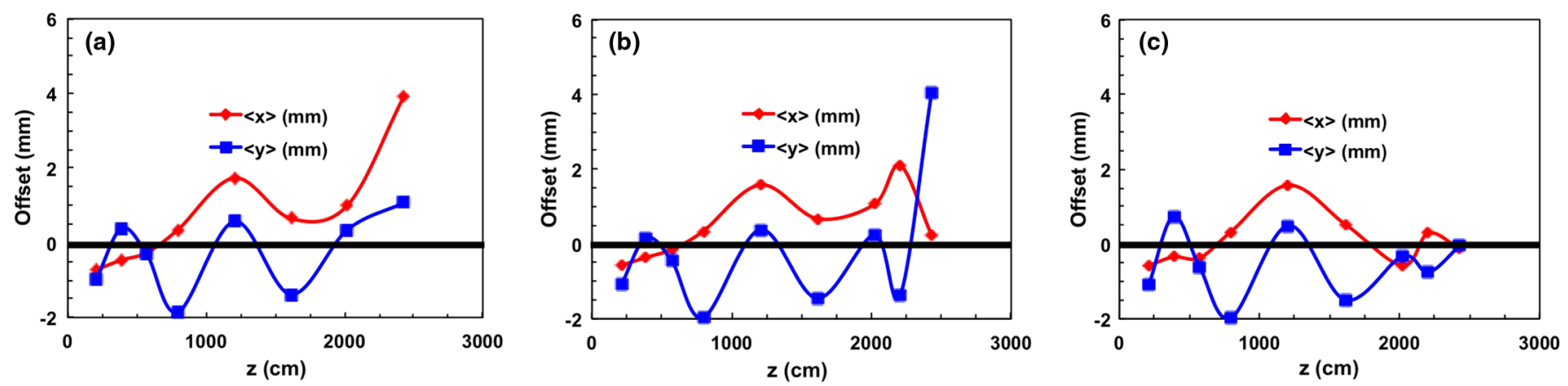

FIG. 7. Measured beam centroid offsets up to BPM14 with (a) all cells on, (b) only first 5 CB on, (c) corrected with dipoles 33( $x, y$ ) and $36(x, y)$ and only first five $\mathrm{CB}$ on. All with new $A(x, y) M(x, y)$ dipole settings.

With this configuration we measured centroid offsets at BPM13 $(z=22.1 \mathrm{~m})$ and after a drift through cells 41-44 at BPM14 $(z=24.3 \mathrm{~m})$ [Fig. 7(b)]. We were also able to extrapolate the angles from the measured centroid offsets at BPM14 and BPM15 assuming a field-free drift (Table II). Beyond BPM15 the beam distribution was $>4.5 \mathrm{~cm}$ and offset $>1 \mathrm{~cm}$, so no centroid measurements could be taken downstream with any confidence due to beam scraping. What is interesting to note is the differences in the centroid position at BPM14 from Figs. 7(a) and 7(b). The centroid at BPM13 was not measured in Fig. 7(a), but it is assumed to be the same. While continuing to operate CB6 the beam continues to be transported slightly off axis in the $y$ direction and largely in the $x$ direction. In the fieldfree drift case, Fig. 7(b), the beam appears to be well centered in $x$ and drifting substantially off axis in the $y$ direction. This is explained by the difference in the betatron motion of the beam centroid of the two cases. There are four magnets between BPMs 13 and 14 and the transport distance is $2.2 \mathrm{~m}$, about $2 / 3 \lambda_{\beta}$ in this region. In Fig. 7(a) the solenoids are operating and the beam has a slight rotational change in $y$ and appears to have a guiding center drift in the $x$ direction, indicating a possible misalignment from the end of CB5 to the beginning of CB6. In Fig. 7(b) the beam just continues to drift with the angle it exits within CB5.

We decided to use dipoles $33(x, y)$ and $36(x, y)$ in cells 33 and 36, which were at the upstream and center of CB5 to perform our alignment procedure. This provided more

TABLE II. Measured beam centroid corrections using dipoles $33(x, y)$ and $36(x, y)$ after five cell blocks at BPM13 $(z=22.1 \mathrm{~m})$ and after a drift through cells $41-44$ at BPM14 $(z=24.3 \mathrm{~m})$.

\begin{tabular}{lcccccc}
\hline \hline & $\begin{array}{c}z \\
(\mathrm{~cm})\end{array}$ & $\begin{array}{c}\langle x\rangle \\
(\mathrm{mm})\end{array}$ & $\begin{array}{c}\left\langle x^{\prime}\right\rangle \\
(\mathrm{mrad})\end{array}$ & $\begin{array}{c}\langle y\rangle \\
(\mathrm{mm})\end{array}$ & $\begin{array}{c}\left\langle y^{\prime}\right\rangle \\
(\mathrm{mrad})\end{array}$ \\
\hline Uncorrected & 17003 & 2206 & 2.10 & -0.83 & -1.37 & 2.41 \\
Corrected & 17019 & 2206 & 0.29 & -0.19 & -0.76 & 0.32 \\
Uncorrected & 17003 & 2430 & 0.24 & -0.42 & 4.03 & 5.73 \\
Corrected & 17019 & 2430 & -0.13 & -0.20 & -0.05 & 0.57 \\
\hline \hline
\end{tabular}

leverage in correction, minimizing the required dipole current. After performing the single dipole iterations and inverting the $4 \times 4$ matrix we were able to correct the beam centroid at both BPM13 and 14 in Fig. 7(c) and Table II.

\section{Cell block 8 steering}

Now that the corrections were made near the middle of the accelerator, we operated CB6-8 and transported the beam to the end of the accelerator. Before we began alignment the centroid was largely offset in CB8 at BPM18, $z=32.4 \mathrm{~m}$ [Fig. 8(a)], and was still large after improving alignment at BPM13 and 14 [Fig. 8(b)]. Again, these offsets are strongly driven by the increased $B$ at the end of the accelerator. At this point it was determined we would use dipoles $57(x, y)$ and $60(x, y)$ in cells 57 and 60 , which were at the upstream and center of CB8 to perform our alignment procedure at BPM19 and 20. After performing the single dipole iterations and inverting the $4 \times 4$ matrix we were able to correct the beam centroid from BPM18-22 as shown in Fig. 8 and Table III. This minimized our centroid offsets to $<2 \mathrm{~mm}$ throughout the whole accelerator.

\section{BBU MEASUREMENTS}

All of the effort described in the previous section was motivated by attempting to reduce the BBU growth throughout the accelerator and possibly gaining a better understanding of the coupling of the beam centroid motion to the BBU. Despite these efforts we were only able to

TABLE III. Measured beam centroid corrections at the end of the accelerator BPM19 $(z=34.3 \mathrm{~m})$ and BPM20 $(z=36.1 \mathrm{~m})$ using dipoles $57(x, y)$ and $60(x, y)$.

\begin{tabular}{lcccrrr}
\hline \hline & Shot & $\begin{array}{c}z \\
(\mathrm{~cm})\end{array}$ & $\begin{array}{c}\langle x\rangle \\
(\mathrm{mm})\end{array}$ & $\begin{array}{c}\left\langle x^{\prime}\right\rangle \\
(\mathrm{mrad})\end{array}$ & $\begin{array}{c}\langle y\rangle \\
(\mathrm{mm})\end{array}$ & $\begin{array}{c}\left\langle y^{\prime}\right\rangle \\
(\mathrm{mrad})\end{array}$ \\
\hline Uncorrected & 17029 & 3432 & 0.01 & 1.40 & 1.25 & 4.20 \\
Corrected & 17049 & 3432 & 0.37 & -0.16 & -0.25 & 0.38 \\
Uncorrected & 17029 & 3614 & 2.56 & 0.07 & 8.91 & 2.37 \\
Corrected & 17049 & 3614 & 0.07 & & 0.45 & \\
\hline \hline
\end{tabular}



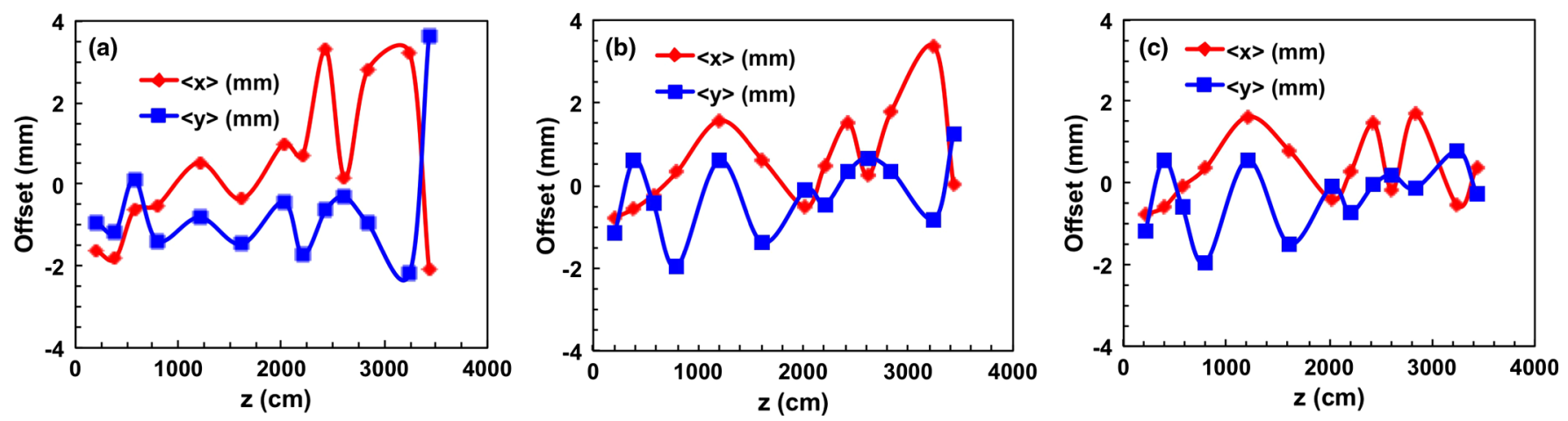

FIG. 8. Measured beam centroid offsets up to BPM19 with (a) nominal dipole settings for a lower field tune, (b) corrected with dipoles $A, M, 33$, and 36 , and (c) corrected with dipoles $A, M, 33,36,57$, and 60 .
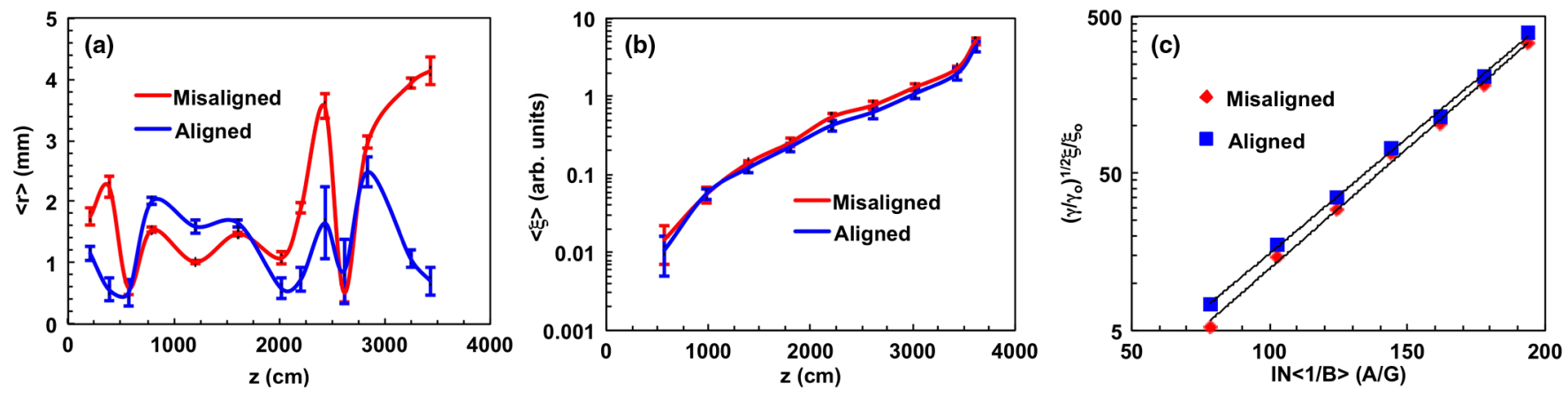

FIG. 9. (a) Measured rms beam centroid offsets, $\langle r\rangle$, (b) measured BBU amplitude $\langle\xi\rangle$, and (c) comparison of BBU growth along the accelerator for the aligned (blue) and misaligned (red) cases. All are averaged over five shots; error bars indicate shot variations.

reduce the rms beam centroid offset along the length of the accelerator (from BPM03-19) to $1.2 \mathrm{~mm}$ from $2.1 \mathrm{~mm}$ for the misaligned case [Fig. 9(a)]. However, we were able to successfully reduce the BBU amplitude $\langle\xi\rangle$ throughout the whole accelerator by an average amount of 19\% [Fig. 9(b)]. Most of the improvement was downstream of BPM07 $(z>10 \mathrm{~m})$ as a slight separation between the two BBU amplitude curves becomes visible in Fig. 9(b). In both cases it is evident that the BBU amplitude increases $>100 \times$ and in order to further reduce the growth a higher field tune was required; these details are outlined in Ref. [22].

Figure 9(c) shows the relative growth of the BBU amplitude for the aligned and misaligned cases. It is worth noting the initial BBU amplitude, $\xi_{o}$, is slightly different for each steering case in Fig. 9(c). A least squares fit was applied to the data sets in Fig. 9(c) and their transverse impedances are indicated in Table IV. The transverse impedance of the aligned case is $4 \%$ less than the misaligned case and each is near the measured and calculated cavity values mentioned in Refs. $[14,22,34,38]$. The final $\langle 1 / B\rangle, \Gamma_{m}$, and minimum and maximum saturation times for these steering cases are also shown in Table IV. Recall the time, $t$, for the BBU to reach maximum growth, $\Gamma_{m}$, or saturate and resonate the induction cell cavity as indicated by [30] is

$$
t=\frac{2 \Gamma_{m} Q}{\omega} .
$$

The measured and calculated $Q$ values for the induction cells from Refs. [14,15,38] range from 3-6 and the frequency range of the peak BBU amplitude is 700$850 \mathrm{MHz}$. The calculated saturation times (Table IV) for the BBU rf oscillations for both steering cases range from 7-19 ns. These results indicate a slight reduction in $\Gamma_{m}$ of $4 \%$ has been achieved with the aligned case. Both growth rates look pretty close, but when examining the slopes or transverse impedances closely it is evident the misaligned case is steeper, leading to a higher transverse impedance and final growth rate.

The BBU growth for the misaligned case along the accelerator is examined more closely with a single-shot

TABLE IV. Calculated transverse impedance of the Axis-I accelerator cells from a least squares fit to the BBU growth data in Fig. 9(c). The final $\langle 1 / B\rangle, \Gamma_{m}$, and the minimum and maximum saturation times for $\Gamma_{m}$ are also tabulated.

\begin{tabular}{lccccc}
\hline \hline & $Z(\Omega / \mathrm{m})$ & $\langle 1 / \mathrm{B}\rangle(1 / \mathrm{G})$ & $\Gamma_{m}$ & $t_{\min }(\mathrm{ns})$ & $t_{\max }(\mathrm{ns})$ \\
\hline Aligned & 1016.3 & $1.04 \mathrm{E}-03$ & 6.6 & 7.4 & 17.9 \\
Misaligned & 1058.3 & $1.04 \mathrm{E}-03$ & 6.8 & 7.7 & 18.7 \\
\hline \hline
\end{tabular}


representation of the average case for five shots, identical to the method described in Ref. [22]. The experimenter observes the increase in the rf oscillations on the raw unintegrated BPM signals for BPMs $7-19(z=9.8 \mathrm{~m}$ to $34.3 \mathrm{~m}$ ) in Fig. 10. Radio-frequency oscillations on the beam envelope are just beginning to become evident at BPM07 [Fig. 10(a)] and saturation is not fully observed until further downstream. Evidence of the BBU saturation is shown at BPM13 [Fig. 10(b)] from a 34 ns window highlighted in red near the head of the beam. The rf oscillations appear to grow in $<20 \mathrm{~ns}$ and then dampen out due to losses in the cells in agreement with the calculations above. This is shown in both the $x$ and $y$ transverse directions of the beam and it increases in amplitude $>3 \times$ at BPM19 in Fig. 10(c). The same saturation and decay is displayed at both BPM13 and BPM19.

There are three additional time slices highlighted through the beam pulse signal in BPM19 [Fig. 10(c)]; they are $18.6 \mathrm{~ns}$ long highlighted in orange, $24 \mathrm{~ns}$ long highlighted in green, and $18.6 \mathrm{~ns}$ long highlighted in blue. Each of these time slices indicates a saturation and decay of the BBU rf oscillations on the beam envelope. Each of these four saturation periods indicates a rise and saturation of the rf oscillations within the calculated times of 7-19 ns above. These independent oscillations are observed consistently on this accelerator providing the BBU instability is present and the growth rate, $\Gamma>4$. Observing the signals on BPM13 [Fig. 10(b)] more closely, the saturation periods are slightly visible here also. However the signature is not as apparent due to the reduced BBU amplitude, which is why the additional saturation periods are not visible upstream at BPM07 [Fig. 10(a)].

Momentum spread leads to a time-dependent beam envelope and time-dependent centroid motion (corkscrew) that could also drive BBU [23-25]. However, the energy spread we have throughout our beam pulse is $\pm 2 \%$ downstream of the injector and typically $<1 \%$ through the accelerator. We also minimize the corkscrew $<200 \mu \mathrm{m}$ minimizing its contribution to the time-dependent BBU we observe in Fig. 10.

The growth rate only increases $30 \%$ from BPM13 to BPM19 [Fig. 9(c)], making it unlikely to observe the convective instability. The convective instability is an increase in the saturation time of the BBU rf oscillations along the accelerator axis without any losses in the cells [39]. We know that we have losses in our cells due to ferrite damping and given the agreement between the measured and calculated saturation times. The convective instability was also not observed on DARHT Axis-II which has a $1.6 \mu$ s pulse $[5,6]$.

Identical saturation effects, like those described above, are seen in the raw signal at BPM20, $z=36.1 \mathrm{~m}$, outside of the accelator lattice. In addition, a specific example of the slight reduction of the BBU amplitude from the aligned case to the misaligned case is to examine the fast Fourier transform (FFT) of the raw signal at BPM20, $z=36.1 \mathrm{~m}$ (Fig. 11). At first glance, the raw $\Delta x$ and $\Delta y$ signals in the top row of Fig. 11 do not show a significant difference.
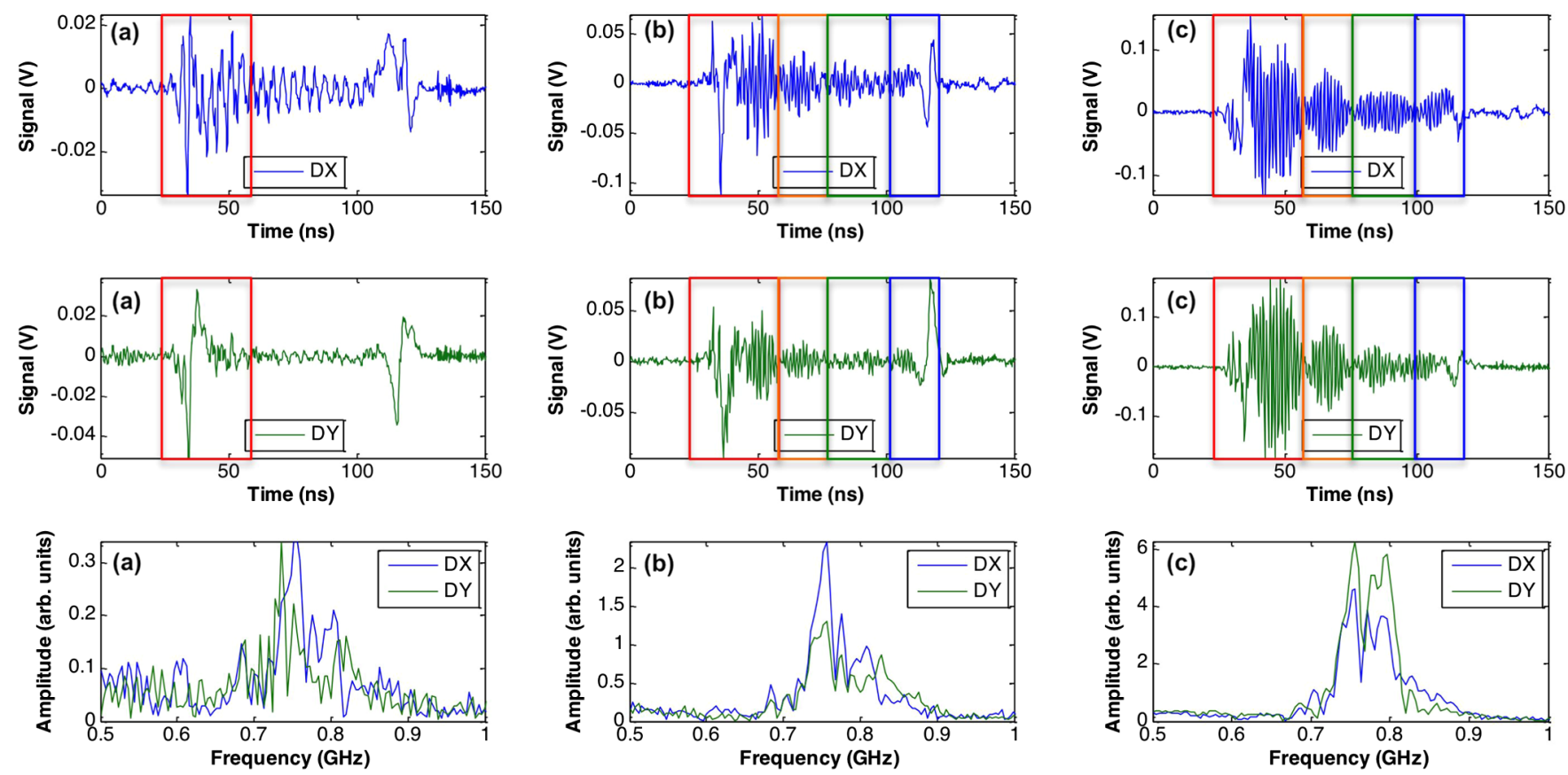

FIG. 10. Top row: unintegrated $\Delta \mathrm{x}$ signals; middle row: unintegrated $\Delta \mathrm{y}$ signals; bottom row: fast Fourier transforms of the signals above to indicate the amplitude and frequency of the BBU on the $2.9 \mathrm{kA}$ beam measured at (a) BPM07 ( $z=9.8 \mathrm{~m}$ ); (b) BPM13 $(z=22.1 \mathrm{~m})$; and (c) BPM19 $(z=34.3 \mathrm{~m})$ (note scale differences). Color coded saturation regions are also shown. 

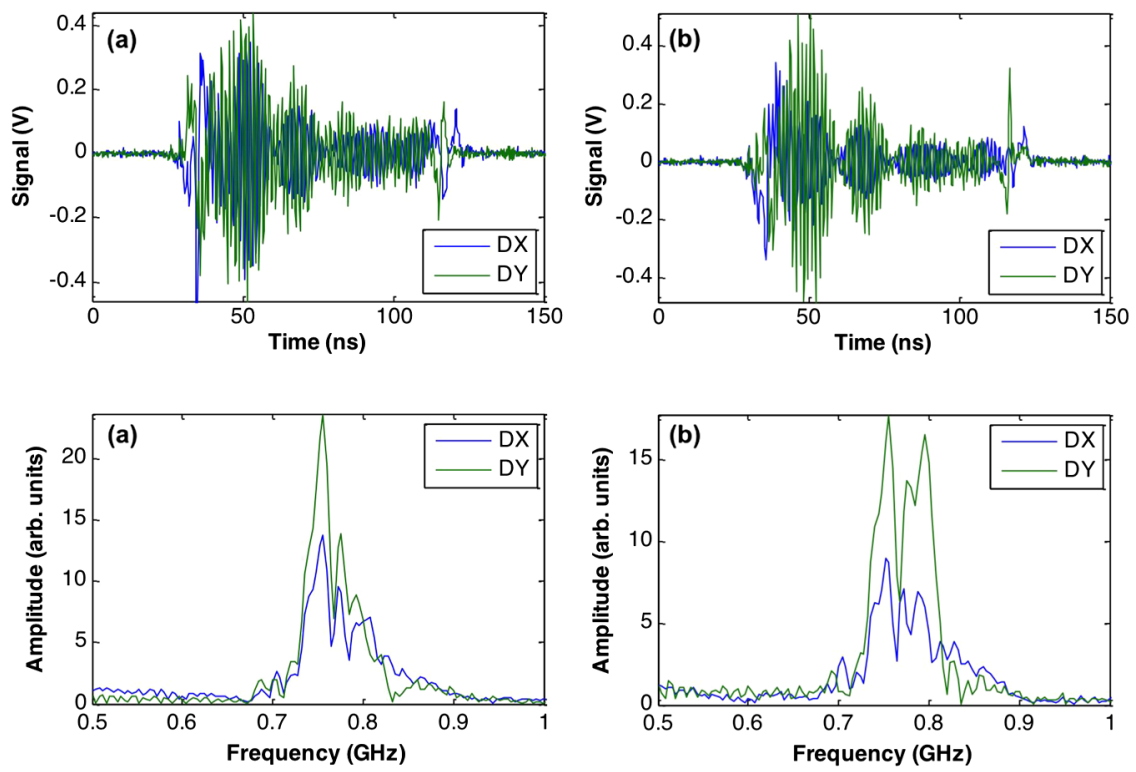

FIG. 11. Top row: unintegrated $\Delta x$ and $\Delta y$ signals measured at BPM20 $(z=36.1 \mathrm{~m})$. Bottom row: fast Fourier transform of the signal above to indicate the amplitude and frequency of the BBU on the $2.9 \mathrm{kA}$ beam for (a) the misaligned case and (b) the aligned case (note scale differences).
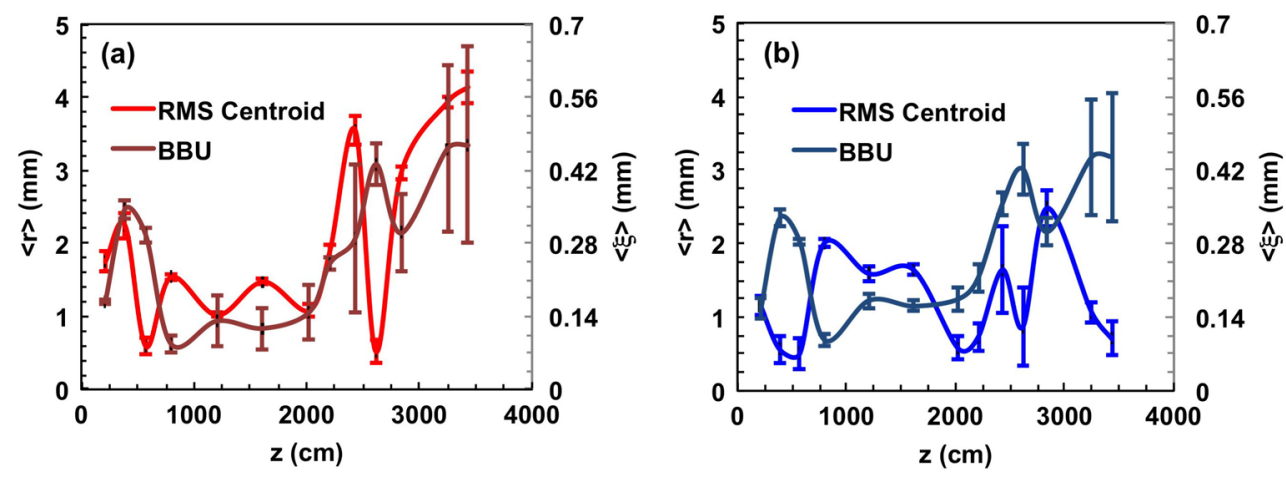

FIG. 12. Measured rms centroid offsets $\langle r\rangle$, and the radial BBU amplitude, $\langle\xi\rangle$, averaged over five shots along the length of the accelerator for the (a) misaligned case (red and dark red) and (b) aligned case (blue and navy blue). Note reduction from (a) to (b).

However, when examining the FFT it is apparent there is $>20 \%$ reduction in the BBU amplitude in both the $x$ and $y$ direction. These waveforms are single-shot representations of the average case for five shots.

It is also interesting to note the comparison of the motion of rms beam centroid, $\langle r\rangle$, versus the average radial BBU amplitude, $\langle\xi\rangle$ (Fig. 12). The curves displayed in Fig. 12 are an average set of five shots with the error bars included. The changes in $\langle r\rangle$ appear to be out of phase with $\langle\xi\rangle$, particularly for the aligned case [Fig. 12(b)]. This is explained by several effects: first the BBU is generated by a transverse magnetic dipole kick due to a misaligned beam, so a reduction in BBU will be seen downstream of a well-aligned beam, as is shown for $z>800 \mathrm{~cm}$ in Fig. 12(b). Second, the angle at which the beam enters the induction cell cavity also plays a roll and it is not measured here. Finally, we are measuring the centroid offsets after increments of four or more cells and not in the actual cells, where the BBU is generated. More than likely the first and last effects contribute to the phase offset we measure.

\section{CONCLUSIONS}

A great deal of effort was made to reduce the beam centroid offsets throughout the accelerator and determine its impact on the BBU instability. This study demonstrates the difficulty of aligning the beam centroid of an intense beam in an induction accelerator. The misalignment contribution from the solenoids is proportional to $B$, which is a function of the beam current transported. A 70\% increase in intensity requires both a comparable increase in field strength for beam transport and therefore at least a $70 \%$ 
larger contribution to beam centroid offsets. Unfortunately due to the lack of quality in the mechanical misalignments of the individual components of the machine, we were only able to reduce the rms centroid motion by $50 \%$.

The results presented provide the first successful demonstration of the reduction of BBU that we know of in an induction accelerator by actively steering the beam with dipoles. These experiments are the first validation, to our knowledge, of the theory in Refs. [30,31], Eqs. (3)-(5) above. A reduction in the rms beam centroid of $50 \%$ throughout the whole accelerator reduced the BBU amplitude by $19 \%$ on average throughout the whole accelerator and the maximum growth rate by $4 \%$. This accelerator was well designed to sample the $\lambda_{\beta}$ for lower field tunes, $B \leq 1 \mathrm{kG}$. However future accelerators, with higher field tunes, $B>1 \mathrm{kG}$, will require closer BPM spacing, although this may not be practical in all cases.

\section{ACKNOWLEDGMENTS}

This work was supported by the National Nuclear Security Administration of the U.S. Department of Energy under Contract No. DE-AC52-06NA25396. I would like to take the opportunity to thank the DARHT Axis-I operators and technicians James Carothers, Sam Snider, Melissa Reed, Jules Carson, Tim McCurdy, Rudy Valdez, and Edward Jacquez for their continued support.

[1] T. O. Raubenheimer, Phys. Rev. ST Accel. Beams 3, 121002 (2000).

[2] K. Ranjan et al., in Proceedings of the 22nd Particle Accelerator Conference, PAC-2007, Albuquerque, NM (IEEE, New York, 2007), p. 2963.

[3] K. Kubo, Phys. Rev. ST Accel. Beams 14, 014401 (2011).

[4] K. Kubo, Phys. Rev. ST Accel. Beams 15, 121002 (2012).

[5] C. Ekdahl et al., IEEE Trans. Plasma Sci. 34, 460 (2006).

[6] C. Ekdahl et al., Phys. Rev. ST Accel. Beams 14, 120401 (2011).

[7] C. A. Ekdahl, Los Alamos National Laboratory, Report No. LA-UR-13-29351, 2013.

[8] M. G. Kelliher and R. Beadle, Nature (London) 187, 1099 (1960).

[9] W. K. H. Panofsky and M. Bander, Rev. Sci. Instrum. 39, 206 (1968).

[10] R. J. Briggs, D. L. Birx, G. J. Caporaso, T. J. Fessenden, R. E. Hester, R. Melendez, V. K. Neil, A. C. Paul, and K. W. Struve, IEEE Trans. Nucl. Sci. 28, 3360 (1981).

[11] G. J. Caporaso, A. G. Cole, and K. W. Struve, IEEE Trans. Nucl. Sci. 30, 2507 (1983).

[12] G. J. Caporaso et al., in Proceedings of 5th International Conference on High-Power Particle Beams, San Francisco, CA, 1983 (IEEE, New York, 1983), p. 427.
[13] G. J. Caporaso, in Proceedings of LINAC, Stanford, CA, 1986, http://www-public.slac.stanford.edu/SciDoc/ docMeta.aspx?slacPubNumber=SLAC-R-303, p. 17.

[14] P. Allison, in Proceedings of the Particle Accelerator Conference, San Francisco, CA, 1991 (IEEE, New York, 1991), p. 520.

[15] P. Allison, in Proceedings of the Particle Accelerator Conference, Vancouver, BC, Canada 1997 (IEEE, New York, 1997), p. 1138.

[16] Y-J Chen, in Proceedings of the Particle Accelerator Conference, 2001 (IEEE, New York, 2001), p. 3490.

[17] C. Tennant et al., in Proceedings of FEL2004 Conference (Comitato Conferenze Elettra, Trieste, Italy, 2004), http:// accelconf.web.cern.ch/AccelConf/f04/, p. 590.

[18] R. Kazimi et al., in Proceedings of the 11th European Particle Accelerator Conference, Genoa, 2008 (EPS-AG, Geneva, 2008), p. 2722.

[19] V. Volkov, Phys. Rev. ST Accel. Beams 12, 011301 (2009).

[20] V. Volkov, J. Knobloch, and A. Matveenko, Phys. Rev. ST Accel. Beams 14, 054202 (2011).

[21] G. J. Caporaso, F. Rainer, W. E. Martin, D. S. Prono, and A. G. Cole, Phys. Rev. Lett. 57, 1591 (1986).

[22] J. E. Coleman , D. C. Moir, C. A. Ekdahl, J. B. Johnson, B. T. McCuistian, G. W. Sullivan, and M. T. Crawford, Phys. Rev. ST Accel. Beams 17, 030101 (2014).

[23] Y.-J. Chen, Nucl. Instrum. Methods Phys. Res., Sect. A 292, 455 (1990).

[24] S. L. Allen et al., in [14], p. 3094.

[25] Y.-J. Chen, Nucl. Instrum. Methods Phys. Res., Sect. A 398, 139 (1997).

[26] S. M. Lund, C. J. Wootton, and E. P. Lee, Nucl. Instrum. Methods Phys. Res., Sect. A 606, 56 (2009).

[27] S. M. Lund et al., in Proceedings of the 23rd Particle Accelerator Conference, Vancouver, BC, Canada, 2009 (IEEE, Piscataway, NJ, 2009), p. 4323.

[28] J. E. Coleman, Ph.D. Thesis, University of California, Berkeley, 2008.

[29] J.E. Coleman et al., Phys. Rev. ST Accel. Beams 11, 050101 (2008).

[30] V. K. Neil, L. S. Hall, and R. K. Cooper, Part. Accel. 9, 213 (1979).

[31] R. J. Briggs et al., Part. Accel. 18, 41 (1985).

[32] Martin Reiser, Theory and Design of Charged Particle Beams (John Wiley and Sons Inc., New York, 1994), pp. 66-76, 210.

[33] Stanley Humphries, Jr., Charged Particle Beams (John Wiley and Sons Inc., New York, 2002), p. 401.

[34] M. Burns et al., in [14], p. 2958.

[35] T. P. Hughes et al., in Proceedings of the Particle Accelerator Conference, Vancouver BC, 1995, (IEEE, Piscataway, NJ, 1995), p. 4323.

[36] P. Allison, Los Alamos National Laboratory, Report No. LA-UR-01-6585, 2001.

[37] K. Takayama and R. J. Briggs, Induction Accelerators (Springer-Verlag Berlin Heidelberg, 2011), p. 137.

[38] L. Walling et al., in [14], p. 2958.

[39] K. Takayama and R. J. Briggs, [37], pp. 150-151. 